Rev. Elev. Méd. vét. Pays trop., 1976, 29 (4) : 323-335.

\title{
La cénurose des petits ruminants d'Afrique centrale Les cénuroses africaines, humaines et animales
}

\author{
par M. GRABER (*)
}

\begin{abstract}
RÉSUMÉ
L'auteur signale la présence au Tchad et en République Populaire du Congo de Coenurus cerebralis (Batsch, 1786) Rudolphi, 1808 dans le tissu conjonctif sous-cutané et intermusculaire, les séreuses et le cerveau du mouton et de la chèvre. Au total, sept cas ont été observés sur les 5000 petits ruminants autopsiés entre 1954 et 1969.

Il étudie les cénuroses animales qui, sur le continent africain, sont dues à tro1s Cestodes parasites de l'intestin des Canidés domestiques et sauvages : Taenia multiceps Leske, 1780, Taenia serialis serialis (Bailliet, 1863) Verster, 1969 n. comb. et Taenia serialis brauni (Setti, 1897) Verster, 1969 n. comb. Leurs affinités sont précisées.

Leurs formes larvaires sont : Coenurus cerebralis des ruminants domestiques et sauvages que l'on trouve non seulement en Afrique du Sud, mais également en Afrique de l'Ouest, de l'Est et du Centre ; Coenurus serialis serialis des Léporidés d'Afrique de l'Ouest, du Sud et du Nord-Est ; Coenurus serialis brauni des Muridés et de certains singes d'Afrique du Nord, d'Afrique du Sud et d'Afrique de l'Est.

La cánurose humaine semble beaucoup plus fréquente que sur les autres continents ( 77 p. 100 des cas mondiaux connus). Elle revêt deux aspects différents : une forme cérébrale à Coenurus cerebralis en Afrique du Sud et vraisemblablement, en Afrique centrale ; une forme sous-cutanée et oculaire à Coenurus serialss brauni en Afrique de l'Est. L'origine des cas rencontrés en Afrique de l'Ouest n'a pu être établie avec suffisamment de précision.
\end{abstract}

Classiquement, la Cénurose se définit comme une affection parasitaire due à la présence dans le cerveau et, plus rarement, dans la moelle épinière de Conurus cerebralis (Batsch, 1786) Rudolphi, 1908, forme larvaire d'un Cestode de la famille des Taeniidae, Taenia multiceps Leske, 1780 , qui, à l'état adulte, vit dans l'intestin de divers Carnivores domestiques et sauvages.

Cette Helminthiase, connue encore sous le nom de "Tournis", affecte principalement les petits ruminants, moutons et chèvres. Elle prend parfois l'allure d'une véritable enzootie dans les troupeaux dont le chien est porteur de Taenia multiceps.

(*) Chaire de Parasitologie. Ecole Nationale Vétérinaire 2, Quai Chauveau, 69337 Lyon Cedex 1.
La maladie a été observée dans de nombreuses régions du globe : Europe, U. R. S. S., Amérique du Nord, Australie.

Dans les pays tropicaux, elle sévit également, mais elle revêt souvent une allure différente comme le montrent plusieurs observations faites en Afrique Centrale au cours de ces 20 dernières années, observations qu'il a paru intéressant de relater, d'autant plus qu'elles ne sont pas sans incidence sur la pathologie humaine de ces régions.

\section{MATERIEL ET MÉTHODE}

780 chèvres et 4200 moutons ont été autopsiés dans toutes les Préfectures du Tchad de 1954 à 1969. 
6 cas de Cénurose ont été découverts se répartissant ainsi :

- chèvre : 1 cas à N'Djaména (ChariBaguirmi) en janvier 1957;

- moutons (*)

- 1 cas à Abécher (Ouaddaï) en janvier 1960 ;

- 2 cas à $\operatorname{Sar}$ (= Fort-Archambault), MoyenChari, en janvier et en avril 1963 ;

- 2 cas à N'Djaména, le premier en novembre 1963 et le second en janvier 1968.

Les autres régions du Tchad paraissent indemnes et, dans ce pays, la Cénurose n'a été décelée ni chez le zébu, ni chez les ruminants sauvages.

En outre, un autre cas de Cénurose a été observé en République populaire du Congo chez un mouton sacrifié à l'abattoir de Brazzaville en 1957.

Les parasites ont été soigneusement recueillis, comptés et mesurés. Un certain nombre d'entre eux ( 2 vésicules chez les moutons examinés à N'Djaména en 1963 et en 1968 ; une vésicule chez le mouton congolais) ont été ouverts et éclaircis au lacto-phénol. Les scolex ont été placés entre lame et lamelle, légèrement écrasés et examinés au microscope, dans le but d'isoler les crochets et d'en apprécier le nombre et la taille.

Parallèlement et par comparaison, ont été étudiés :

- un Cénure prélevé dans l'encéphale d'un mouton mort en 1959 à l'Ecole nationale vétérinaire de Lyon;

- des Cénures de lapin provenant des alentours de Lyon ;

- des Taenia serialis récoltés dans l'intestin d'un chien de même origine ;

Soit, au total, 112 scolex et 1553 crochets dont :

- Cénure du mouton de Brazzaville : 143 grands crochets et 158 petits;

— Cénure des moutons du Tchad : 271 grands crochets et 273 petits ;

- Cénure du mouton français : 146 grands crochets et 146 petits ;

- Cénure du lapin : 173 grands crochets et 184 petits ;

(*) Surtout des Bororos.
- Taenia serialis du chien : 17 grands crochets et 42 petits.

\section{RÉSULTATS}

1. Du viyant de l'animal, la Cénurose se traduit par des tuméfactions de dimension variable, plus ou moins arrondies, froides, indolores et remplies d'un liquide sous tension.

Elles siègent en différents points du corps, surtout au niveau de l'épaule et de la cuisse, mais on en trouve également sur la paroi thoracique, en arrière du scapulum, dans la région maxillaire et sous le cou. Elles ne descendent ni au-dessous du jarret, ni au-dessous du genou.

Quand elles sont nombreuses et volumineuses - ce qui est souvent le cas - -, l'animal prend un aspect "boursouflé » caractéristique avec de véritables pseudo-tumeurs qui pendent latéralement sur les côtés du corps ou en arrière, au-dessus du creux poplité qu'elles recouvrent.

Le mouton atteint présente des troubles de la locomotion : la marche est gênée, raide, ralentie, avec, parfois, des boiteries lorsque le Cénure comprime le nerf sciatique. Fréquemment, l'animal se couche en position de chien assis.

Dans ces conditions, il suit mal le troupeau, se nourrit moins bien, d'où amaigrissement et anémie.

2. A l'autopsie, on retrouve ces «kystes》 logés surtout dans le tissu conjonctif souscutané et dans le conjonctif intermusculaire de l'épaule et de la cuisse, plus rarement dans le foie, le poumon, le rein, le cœur, le péritoine, les ganglions lymphatiques, le diaphragme, exceptionnellement dans le cerveau.

Sur les 7 cas observés en Afrique centrale, on dénombre :

- localisations intermusculaires seules: 4 ovins (*). En général, de 1 à 6 parasites ;

- localisations intermusculaires et cérébrales : 1 mouton. 15 kystes dont 1 à la base du cerveau ;

- localisation péritonéale : 1 chèvre. 1 seul kyste ;

- Cénurose généralisée à tous les organes

$\left(^{*}\right)$ Dont le mouton de Brazzaville (un parasite sous les adducteurs de la cuisse). 
(sauf le cerveau) : plus de 25 kystes chez un mouton de N'Djaména ;

Ils mesurent $5,5-11 \times 3,5-10 \mathrm{~cm}$ (moyenne sur 16 kystes, $7,8 \times 6,2 \mathrm{~cm}$ ).

Ils sont entourés d'une membrane conjonctive blanche et opaque $\left({ }^{*}\right)$ (P. I, fig. 1), fortement adhérente aux tissus adjacents, ce qui rend leur isolement difficile. Ce fait confirme des observations antérieures (15).

\section{Le parasite}

L'ouverture de la lésion libère une vésicule flasque, remplie d'un liquide clair, translucide

(*) D'où confusion possible avec des lésions d'Echinococcose. et enveloppée d'une paroi mince à l'intérieur de laquelle sont insérées de nombreuses taches blanchâtres $(* *)$ punctiformes et groupées en plages irrégulières (PI. I, fig. 2 et Pl. II et III, fig. 1 et 2). Chacune de ces taches correspond à une invagination céphalique au fond de laquelle est logé le scolex.

Chaque plage est formée d'un nombre variable de scolex :

- vésicule du mouton de Brazzaville (Pl. III, fig. $2: 25$ plages de 15 à 29 scolex (moyenne : 19) ;

(**) Certaines sont capables de se détacher et de tomber dans le liquide, ce qui les a fait prendre - à tort - pour des vésicules-filles (15).
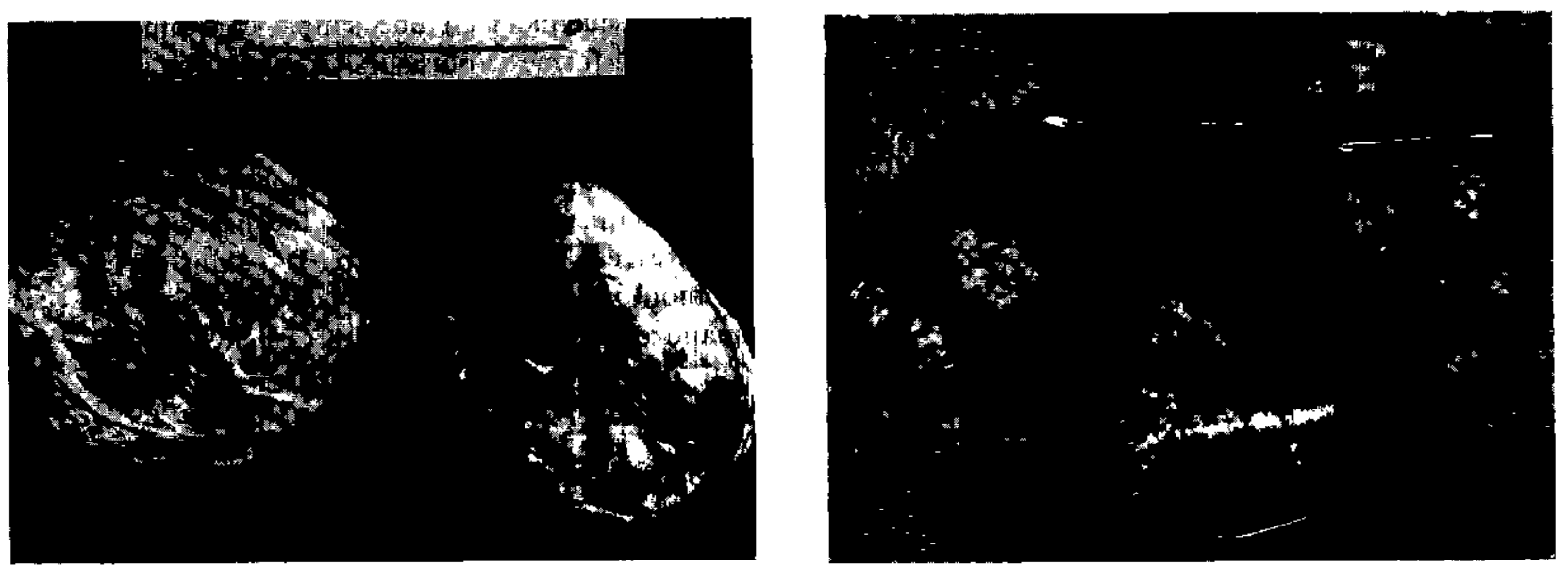

PLANCHE No 1

Figure $n^{\circ} 1$. - Lésions de Cènurose entourées de leur adventice

Figure $\mathrm{n}^{0}$ 2. - Vésicule de Coenurus cerebralis sans son adventice (mouton N'Djaména).
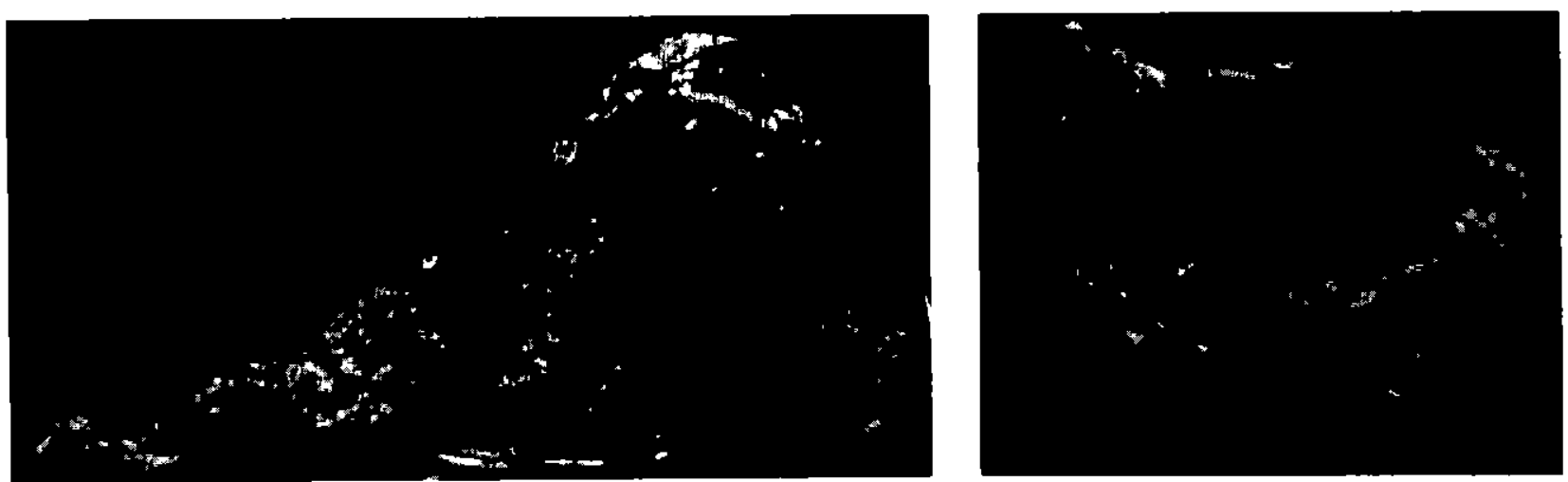

PLANCHE NO II

Figure $\mathbf{n}^{\circ}$ 1. - Vésicule de Coenurus cerebralis ouverte (mouton Brazzaville).

Figure $n^{0} 2$. - Vésicule de Coenurus cerebralis ouverte-Cerveau (mouton Lyon). 

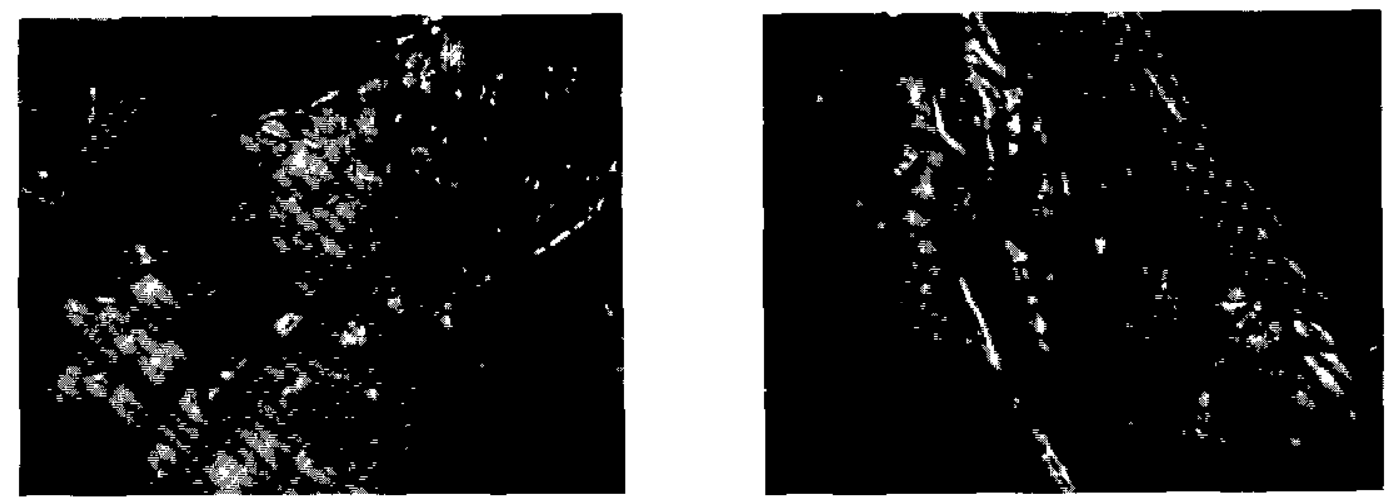

PLANCHE No III

Figure $n^{\prime}$ 1. - Scolex sur la paroi de la vćsicule (mouton N'Djaména)

Figure $n^{\prime 2}$ 2. - Scolex sur la paroi de la vésicule (mouton Brazzaville)

- vésicule du mouton de France: 15 plages de 28-36 scolex ;

- vésicules des moutons du Tchad (Pl. III, fig. 1) : 27 à 47 plages de 16 à 52 scolex (moyenne, 24).

Les scolex dont les dimensions sont données au tableau $\mathrm{N}^{\circ} 1$ mesurent 0,76 à $1 \mathrm{~mm}$ de diamètre. Ils portent 4 ventouses de $240-340 \times$ 240-320 $\mu$ et un rostellum de petite taille $(360-460 \times 320-460 \mu)$ sur lequel est implantée une double couronne de $26-32$ crochets (PI. IV, fig. 1 et 2).

Les crochets de la première rangée, les plus grands (PI. V, fig. 1 et 2, Pl. VI, fig. 1), sont longs de $152-184 \mu$ (en moyenne, 171,3 $\mu$ pour 414 crochets des Cénures du Tchad et du Congo). La distance de la pointe de la lame au sommet de la garde (AB) est inférieure à la distance du sommet de la garde à l'extrémité postérieure du manche (BC). La lame est assez fortement courbée. La garde coniforme possède sur son bord antérieur une petite protubérance. Le manche, légèrement élargi dans sa partie moyenne et dont l'extrémité est régulièrement arrondie, montre, sur le bord dorsal, une saillie située en son milieu ou un peu en avant.

Les crochets de la seconde rangée, les plus petits (Pl. V, fig. 1 et 2, Pl. VI, fig. 1), sont longs de $104-132 \mu$ (moyenne $113 \mu$ pour 431 crochets des Cénures du Tchad et du Congo). Les distances $\mathrm{AB}$ et $\mathrm{BC}$ sont, à peu de choses près, égales. La lame est légèrement courbée. La garde est relativement large, d'aspect réniforme ; ce qui la fait paraître bifide, alors qu'elle ne l'est pas réellement. L'extrémité postérieure du manche est arrondie et toujours incurvée dorsalement.
Un certain nombre de scolex présentent des anomalies qui portent sur le nombre de crochets (2 à 14 seulement en une simple couronne) et sur leur forme: plusieurs d'entre eux sont dépourvus de manche; d'autres n'ont ni garde, ni manche; certains même sont devenus presque punctiformes (Pl. VI, fig, 2). De telles anomalies sont fréquentes lorsque la localisation des Cénures est cérébrale : 20 p. 100 dans le cas du mouton sacrifié à Lyon $(*)$, contre 3,7 p. 100 quand elle est intermusculaire (Tchad, Congo).

\section{Discussion}

4.1. La lecture du tableau $\mathrm{N}^{0}$ I montre que les Cénures intermusculaires des moutons du Tchad et de la République populaire du Congo ne diffèrent pas fondamentalement des Cénures cérébraux que l'on trouve dans l'encéphale des ovins élevés dans les zones tempérées (France) : l'aspect du scolex, la forme des crochets et leurs dimensions sont à peu près les mêmes. Il s'agit donc bien de Coenurus cerebralis (Batsch, 1786) Rudolphi, 1808, forme larvaire d'un Taenia des carnivores domestiques et sauvages, Taenia ( $=$ Multiceps) multiceps Leske, $1780\left(^{* *}\right)$.

La Cénurose sous-cutanée et intermusculaire des ruminants domestiques est bien connue. Signalée pour la première fois aux Indes, il y a déjà 70 ans (13), elle a été revue, depuis, à de nombreuses reprises :

- dans le sous-continent Indien, au Pakistan, au Bengladesh et au Sri-Lanka $(1,4)$;

(*) En France (Savoie), les Cénures cérébraux de chamois présentent le même pourcentage d'anomalies. (**) Selon la nomenclature d'ESCH et SELF (8) et de VERSTER (27). 

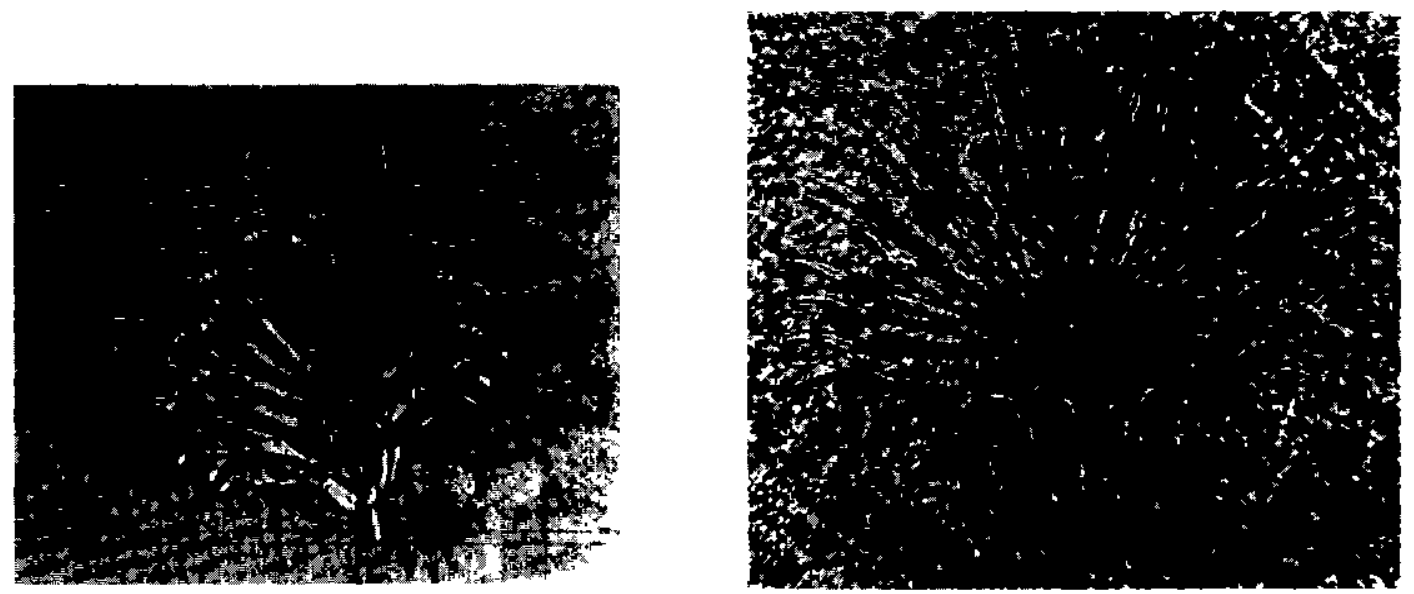

PLANCHE No IV

Figure $n^{\circ} 1$. - Scolex de Coenurus cerebralis $(\times 130)$ (mouton N'Djaména)

Figure $n^{\circ} 2$. - Scolex de Coenurus cerebralss $(\times 210)$ (mouton France)
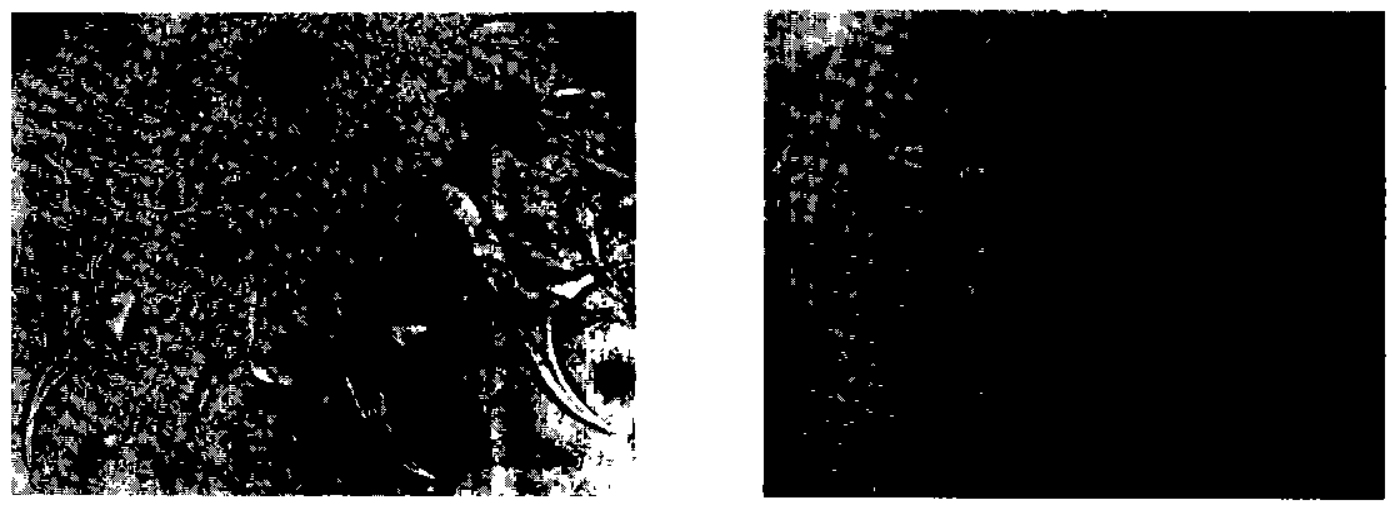

PLANCHE No $\mathrm{V}$

Figure $n^{\circ}$ 1. - Grands et petits crochets ( $: 250$ ) (mouton N'Diaména).

Figure $n^{\circ} 2$. - Grands et petits crochets ( $\times 250$ ) (mouton Brazzaville).
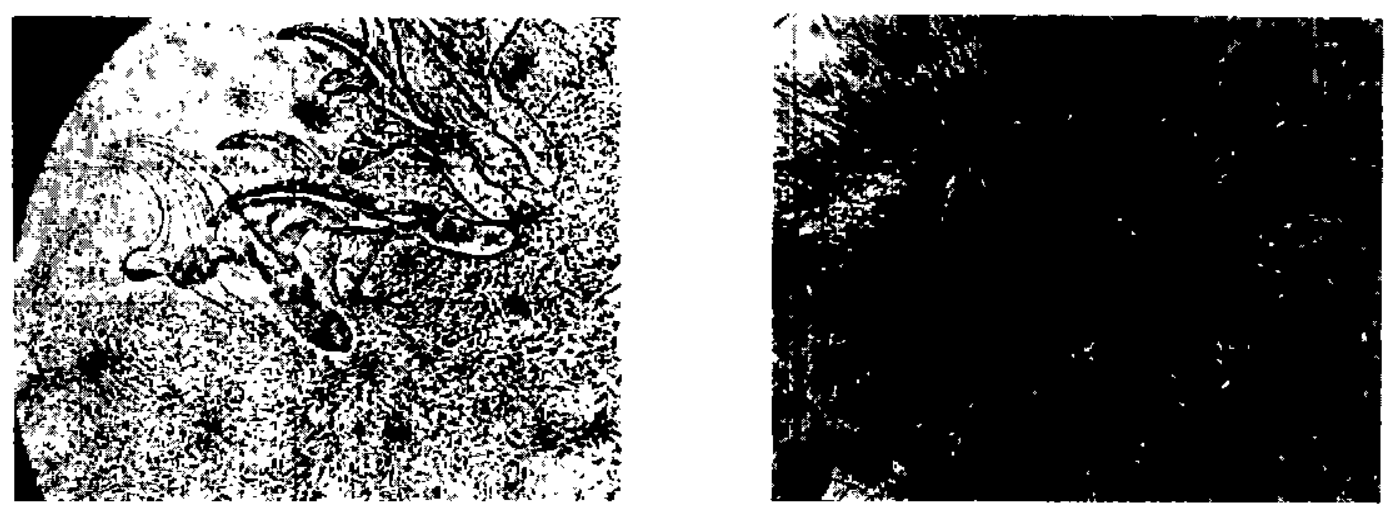

PLANCHE No $\mathrm{Vl}$

Figure $n^{\circ}$ 1. - Grands et petits crochets ( $\times 250$ ) (mouton France).

Figure $n^{\circ} 2$. - Scolex anormal (mouton France) 
TABLEAU $\mathrm{N}^{\circ}$ I - Dimensions des scolex et des crochets examinés

\begin{tabular}{|c|c|c|c|c|c|c|}
\hline \multirow{2}{*}{$\begin{array}{l}\text { Paragites } \\
\text { et origine }\end{array}$} & \multirow{2}{*}{$\begin{array}{l}\text { Scolex } \\
\text { (en an) }\end{array}$} & \multirow{2}{*}{$\begin{array}{l}\text { Nombre de } \\
\text { crochets }\end{array}$} & \multicolumn{2}{|c|}{ Grands crocbets } & \multicolumn{2}{|c|}{ Petits crochets } \\
\hline & & & $\begin{array}{r}\text { Limites } \\
(\text { en } \mu)\end{array}$ & $\begin{array}{c}\text { Moyenne } \\
(\text { en } \mu)\end{array}$ & $\begin{array}{r}\text { Limites } \\
(\text { en } \mu)\end{array}$ & $\begin{array}{l}\text { Moyenne } \\
\text { (en } 11)\end{array}$ \\
\hline $\begin{array}{l}\text { C. cerebralis } \\
\text { Moutons }\end{array}$ & & & & & & \\
\hline France & $0,74-I$ & $24-32$ & $150-172$ & 169,5 & $96-120$ & 115 \\
\hline Tchad & & & & & & \\
\hline 1963 & $0, B 0-I$ & $28-32$ & $152-184$ & 172,7 & $108-132$ & 116,1 \\
\hline 1968 & $0,76-I$ & $26-32$ & $160-180$ & 171,4 & $104-120$ & 112,9 \\
\hline Brazzaville & $0,80-I$ & $26-32$ & $156-180$ & 170 & $100-125$ & 111,1 \\
\hline Total & $0,76-I$ & $26-32$ & $152-184$ & 171,3 & $104-132$ & 113 \\
\hline Taenia semalis & & & & & & \\
\hline Lapin & $0,66-0,8$ & $26-30$ & $140-160$ & 152,2 & $82-108$ & 96,6 \\
\hline Chren & $0,66-1,2$ & $26-30$ & $140-152$ & 142 & $88-104$ & 96,3 \\
\hline Total & $0,66-1,2$ & $26-30$ & $140-160$ & 151,3 & $82-108$ & 96,4 \\
\hline
\end{tabular}

- en Asie centrale (1);

- en Europe : Albanie ;

- en Afrique $(10,17,19)$.

Selon ABULADZE (1), les parasites en cause sont Taenia (= Multiceps) gaigeri Hall, 1916 pour les Cestodes des zones tropicales et Taenia (= Multiceps) skrjabini Popov, 1937 pour ceux du Kazakstan (Asie centrale). Les dimensions comparatives du scolex, le nombre de crochets et leur taille sont donnés au tableau $\mathrm{N}^{\circ}$ II.

Comme l'on peut s'en rendre compte, ce sont des Taeniidae extrêmement voisins qu'il est difficile de séparer les uns des autres. Actuellement, certains auteurs maintiennent cette distinction. D'autres, au contraire, estiment que Taenia gaigeri est synonyme de Taenia multiceps, notamment NAGATY et EZZAT (17): ces auteurs examinent des Cénures récoltés dans le

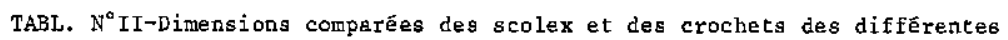
formes de Taenia multiceps

\begin{tabular}{|c|c|c|c|c|}
\hline Parasites et auteurs & $\begin{array}{l}\text { Scolex } \\
\text { (en mm) }\end{array}$ & $\begin{array}{l}\text { Nombre de } \\
\text { crochets }\end{array}$ & $\begin{array}{l}\text { Grands crochets } \\
\text { Limites et moyenne } \\
\text { (en } \mu)\end{array}$ & $\begin{array}{c}\text { Petits crochets } \\
\text { Limites et moyenne } \\
(\text { en } \mu)\end{array}$ \\
\hline $\begin{array}{l}\text { Taenia gaigem } \\
\text { Hal1 (40) } \\
\text { Bhaduri et Maplestone (9) } \\
\text { Claphan (19) } \\
\text { Nagaty et Ezzat (58) } \\
\text { Mathur et Dutt (50) }\end{array}$ & $\begin{array}{c}0,95 \\
0,69-1,2 \\
- \\
- \\
-\end{array}$ & $\begin{aligned} 28 & -32 \\
26 & -34 \\
& - \\
24 & -32 \\
28 & -30\end{aligned}$ & $\begin{array}{c}150-180 \\
144-16 \mathrm{~B} \\
145(15989) \\
144-19 \mathrm{~B} \\
152-169 \\
(164)\end{array}$ & $\begin{array}{r}115-150 \\
84-124 \\
103-160 \\
103-135 \\
114-118 \\
(117)\end{array}$ \\
\hline $\begin{array}{l}\text { Taenia akrjabini } \\
\text { Popov (63) } \\
\text { Boev (12) }\end{array}$ & $\begin{array}{c}0,75-0,9 \\
-\end{array}$ & $\begin{array}{c}32 \\
26-34\end{array}$ & $\begin{array}{c}150 \\
151-167\end{array}$ & $\begin{array}{c}110 \\
99-118\end{array}$ \\
\hline $\begin{array}{l}\text { Taenia muitiaeps } \\
\text { Abuladze (I) } \\
\text { Jayeux et Baer (45) } \\
\text { Ranson (66) } \\
\text { Dollfus (24) } \\
\text { Clapham (19) } \\
\text { Verster (95) }\end{array}$ & $\begin{array}{c}0,8 \\
0,75 \\
- \\
0,75-0,95\end{array}$ & $\begin{array}{c}22-32 \\
2 B \\
- \\
22-30\end{array}$ & $\begin{array}{c}150-170 \\
147-152 \\
120-170 \\
(142,2) \\
157-177 \\
(167,2)\end{array}$ & $\begin{array}{r}90-130 \\
87,7-95 \\
76-130 \\
98-136 \\
(125,5)\end{array}$ \\
\hline
\end{tabular}


tissu conjonctif sous-cutané et intermusculaire d'un Ibex de Nubie, d'un hybride d'Ibex et de chèvre mort au jardin zoologique de Giza et d'un mouton originaire du Soudan. Ils arrivent à la conclusion que Taenia gaigeri et Taenia multiceps sont semblables et que les différences (crochets, position des testicules, poche du cirre, vitellogène) observées entre les deux parasites (15) sont sujettes à d'amples variations (*). Il est donc impossible de prendre en considération de tels critères pour différencier les deux espèces.

Par ailleurs, HALL (15) insiste sur le fait que l'hôte intermédiaire normal de Taenia multiceps est le mouton: dans cette espèce, parmi les embryons de Taenia distribués dans tout l'organisme par la circulation générale, seuls, ceux qui parviennent dans les centres nerveux, se développent, les autres dégénèrent. Par cortre, Taenia gaigeri évolue surtout chez la chèvre et les embryons se localisent non seulement dans le système nerveux central, mais encore dans le conjonctif sous-cutané ou intermusculaire et dans les séreuses.

En réalité, cette distinction n'est qu'apparente, car, si, chez la chèvre, la Cénurose est, la plupart du temps, intermusculaire $\left({ }^{* *}\right)$, chez le mouton, on connaît de nombreux cas extracérébraux $(14,17,19)$. L'argument de HALL (15) tombe donc de lui-même.

Récemment, VERSTER (27) réexamine les exemplaires originaux de Taenia gaigeri, des Cestodes adultes de la même espèce recueillis en Egypte chez un chien expérimentalement infesté, des Taenia multiceps récoltés en Afrique du Sud dans l'intestin du chacal et du chien, ainsi que des Cénures de mouton de même origine. Pour elle, il n'existe qu'une seule espèce, Taenia multiceps, Taenia skrjabini ne représentant qu'une sous-espèce adaptée aux conditions bien particulières de l'Asie centrale soviétique.

4.2. Un autre point mérite de retenir l'attention: celui des relations entre Taenia multiceps et Taenia serialis. Celui-ci qui, à l'état adulte, vit dans l'intestin du chien et de divers Canidés, évolue chez les Lagomorphes (lapins et lièvres), exceptionnellement chez certains Rongeurs (écureuil, chinchilla). CLA-

(*) Signalées dès 1940 par BHADURI et MAPLESTONE (4).

(**) Chez les bovins, la localisation intermusculaire est rarissime : elle n'a été observée qu'une seule fois en U. R. S. S.
PHAM $(6,7)$, après avoir examiné 225 scolex pris au hasard et mesuré plus de 1000 crochets, estime que les 2 cestodes ne peuvent être distingués sur de simples critères morphologiques : il n'existe qu'une seule espèce largement répandue, Taenia multiceps $\left({ }^{*}\right)$, présentant des «souches» ou «races" adaptées soit aux centres nerveux des Ongulés, soit au tissu conjonctif intermusculaire des Léporidés. Cette opinion est partagée, avec plus ou moins de réserve, par plusieurs auteurs. ESCH et SELF (8), entre autres, infestent des souris à partir d'oncosphères de Taenia serialis. Ils obtiennent des Cénures localisés à presque tous les tissus et tous les organes, y compris le cerveau. Ils en concluent que le parasite a une grande facilité d'adaptation et que le développement de la forme larvaire dépend plus de l'hôte que du Cestode lui-même.

Malheureusement, cette façon de voir est loin de faire l'unanimité et la plupart des auteurs maintiennent la dualité des espèces $(1,27)$ en se basant :

4.2.1. Sur des critères morphologiques: La longueur des crochets $(* *)$ de Taenia et de Coenurus serialis, telle qu'elle figure au tableau $\mathrm{N}^{\circ} 1$, est inférieure à celle des crochets de Coenurus multiceps: $151,3 \mu$ contre $169,5 \mu$ $(-16$ p. 100) et $96,4 \mu$ contre $115 \mu$ ( -11 p. 100). Ces chiffres correspondent à peu près à ceux donnés par NAGATY et EZZAT (17). Toutefois, ce n'est pas une règle absolue et, souvent, la longueur maximale des petits crochets recoupe la longueur minimale des grands.

On a insisté (17) également sur la présence, en arrière de la garde et sur le côté ventral des petits crochets, d'une protubérance qui serait caractéristique. L'examen à Lyon de 226 petits crochets de Taenia serialis n'a pas permis de confirmer la validité de ce caractère qui est inconstant ( 10 p. 100 des cas environ).

Plus sérieux paraît être le critère retenu par VERSTER (27): dans le proglottis mûr, le vagin de Taenia serialis est entouré d'un sphincter complet de 36-59 $\mu$, alors que, chez Taenia multiceps, il s'agit d'un simple bourrelet de fibres musculaires (14-23 $\mu$ ), incomplet et unilatéral. Les exemplaires de Taenia serialis examinés à Lyon avaient tous un sphincter vaginal complet, mais ce caractère ne peut

(*) En vertu de la loi de priorité.

(**) Les plus grands surtout (7). 
être observé qu'après coloration parfaite du parasite, ce qui n'est pas toujours réalisable.

4.2.2. Sur des critères anatomiques: disposition linéaire des scolex sur le Cénure de Taenia serialis et présence constante d'une adventice, tandis que, chez Coenurus cerebralis, la disposition des scolex est irrégulière et l'adventice fait défaut. Là encore, ces critères sont sujets à variations et ne peuvent donc être pris en considération.

4.2.3. Sur des critères biologiques $\left({ }^{*}\right)$ : A de nombreuses reprises, on a tenté d'infester des lapins à partir d'oncosphères de Taenia multiceps ou de Taenia skrjabini provenant de chiens ayant reçu les Cénures correspondants : les essais sont tous demeurés infructueux.

Réciproquement, au siècle dernier, BAILLIET (2) a cherché à infester des moutons à partir de Taenia serialis, sans succès d'ailleurs. En 1969, au Sénégal, GRETILLAT (**) (21) reproduit cette expérience. Il recueille chez des Léporidés tués entre Dakar et M'Bao des Cénures sous-cutanés appartenant à l'espèce Coenurus serialis, Cénures qu'il fait absorber à des chiens neufs. Au bout d'un certain temps, ceux-ci éliminent des anneaux de Taenia serialis. Les chiens sont alors sacrifiés et les segmonts gravides sont donnés à 3 moutons de race locale. A l'autopsie, quelques mois plus tard, aucun Cénure n'a été rencontré ni dans le cerveau, ni dans le conjonctif intermusculaire.

Il est donc évident que, jusqu'à preuve du contraire, Taenia multiceps et Taenia serialis, bien que très voisins, ne peuvent être confondus en une seule et même espèce.

Taenia serialis comprend, selon VERSTER (27), deux sous-espèces :

- Taenia serialis serialis $(=T$. antarctica $=T$. packi $=T$. laruei ) dont il vient d'être question.

- Taenia serialis brauni qui a été décrite pour la première fois par SETTI à partir de 20 Cestodes récoltés dans l'intestin d'un chien sacrifié en Erythrée (Ethiopie). Le cycle évolutif a été découvert par Von LINSTOW en Egypte, puis redécrit au Zaïre par FAIN $(11,12)$.

L'hôte intermédiaire est un Rongeur appartenant aux familles des Muridés (Dendromus,

(*) Coenurus serialis, à la dıfférence de Coenurus c'erebralis, est capable de donner des vésicules-filles, externes ou internes, semblables à la vésicule-mère.

$\left({ }^{*}\right)$ Communication personnelle.
Lemniscomys, Praomys, Rattus, Otomys, Mus), des Gerbillidés (Gerbillus), des Rhyzomyidés (Tachyoryctes) et des Hystricidés (Hystrix). Les Léporidés, les Ruminants et le Cobaye sont totalement réfractaires à l'infestation.

Les localisations larvaires sont assez anarchiques : on rencontre habituellement Coenurus brauni dans le tissu conjonctif sous-cutané, parfois dans la cavité thoracique ou abdominale, plus rarement dans le cerveau.

Dans cette sous-espèce, est inclus (27) également Taenia glomeratus, parasite du chien à l'état adulte (5) et, à l'état larvaire, de la souris et des Gerbilles (5) Coenurus otomys (6) et Coenurus spalacis des rats-taupes (Tachyoryctes) d'Ethiopie, du Natal et du Kenya (I) pourraient aussi appartenir à cette sous-espèce. Toutefois, le Taenia adulte est inconnu: elles demeurent donc à l'état de species inquirendae.

Le scolex de Taenia serialis brauni mesure 0,7-1,5 mm. Il porte un rostellum armé d'une double couronne de 26-34 crochets dont la longueur varie de 125 à $160 \mu$ pour les plus grands et de 80-114 $\mu$ pour les plus petits. La garde des petits crochets est bilobée.

\section{COMMENTAIRES}

1. Il est frappant de constater que la Cénurose du mouton présente le même aspect dans les savanes africaines et dans certaines steppes de l'Asie centrale soviétique. Qui est responsable de cette situation : l'hôte ou le parasite ?

2 hypothèses viennent à l'esprit :

- ou la Cénurose du mouton est essentiellement une affection du système nerveux central. Dans ce cas, les localisations sous-cutanées ou intermusculaires sont le fait de "variétés », voire de sous-espèces de Taenia multiceps, mieux adaptées à ces tissus ;

- ou la Cénurose des petits ruminants est primitivement - comme celle des Léporidés et des Rongeurs sauvages - une affection du tissu conjonctif sous-cutané ou intermusculaire. Dans certaines circonstances non précisées, les réactions de l'hôte parviennent à éliminer la plupart des éléments infestants, à l'exception de ceux qui atteignent les vaisseaux irriguant les centres nerveux.

Il est difficile, dans l'état actuel de nos connaissances, de trancher et, seules, des expériences poussées permettront d'y voir plus clair. 
2. En ce qui concerne la répartition géographique des Cénuroses animales sur le continent africain, on en est resté, jusqu'à présent, aux conclusions de NELSON, PESTER et RICKMAN (18), c'est-à-dire :

- «either Taenia multiceps nor Taenia serialis has been found in East african Carnivores. "

- «In North and South Africa both Taenia serialis and Taenia multiceps occur ; in between, in East, West and Central Africa, there are probably several intermediate forms. "

10 ans plus tard, la situation est la suivante :

2.1. Taenia multiceps et son Cénure ont été signalés en Egypte, au Soudan $\left(^{*}\right)(10,17$, 19), en Ethiopie (3), au Kenya où le parasite a été recueilli dans le cerveau du mouton, de la chèvre et du porc $(20,23)$, en Afrique du Sud avec un taux d'infestation de 0,8 p. 100 dans certains troupeaux de moutons, en Angola (16), au Tchad, en République populaire du Congo et au Sénégal (21) où l'affection semble rare chez les Ovins.

2.2. Taenia serialis serialis et son Cénure ont été rencontrés en Egypte, en Ethiopie où 30 p. 100 des Léporidés hébergent ce Cestode (3), en Afrique du Sud (27), en Angola (16), au Dahomey, au Ghana (6) et au Sénégal où un Léporidé sur 3 est atteint (21). Nous l'avons observé également à plusieurs reprises sur des lièvres du Tchad.

2.3. Taenia serialis brauni et son Cénure paraissent plus fréquents dans l'Est du continent africain : Ethiopie, Kenya, Uganda, Ruanda et République démocratique du Congo $(11,12)$. Le parasite existe aussi en Egypte, en Tunisie, en Afrique du Sud (27) et, peut-être, au Nigeria (voir infra).

L'aire de répartition de ces 3 Taeniidae couvre donc la plus grande partie du continent africain. Taenia multiceps, en particulier, sera d'autant plus abondant que les moutons, hôtes intermédiaires, seront plus nombreux.

3. L'hôte définitif principal de Taenia multiceps, de Taenia serialis serialis et de Taenia serialis brauni est le chien. Les Carnivores sauvages jouent également un rôle important

(*) L'identification de MAGZOUB (Coenurus serialis) basée uniquement sur le diamètre de la vésicule est certainement erronée. L'examen des trois figures montre qu'il s'agit, selon toute vraisemblance, de Coenurus cerebralis. dans la transmission des Cénuroses, notamment le chacal chez lequel 7 à 27 p. 100 des Cénures de Taenia multiceps donnent naissance à des Cestodes adultes. En Afrique, ce Canidé héberge souvent l'un ou l'autre Cestode. Taenia serialis serialis a également été trouvé chez l'hyène en Zambie.

4. A la liste des hôtes intermédiaires donnée plus haut, il convient d'ajouter :

4.1. En ce qui concerne Coenurus cerebralis, un certain nombre de Ruminants sauvages originaires d'Afrique, autopsiés dans les parcs zoologiques européens au cours du siècle dernier (14) : Alcelaphus sp., des gazelles et des antilopes en France; L'antilope rouanne, Hippotragus equinus et une gazelle dorcas Gazella dorcas, en Allemagne.

Plus récemment, des Cénures ont été mis en évidence :

- en Egypte, chez l'ibex de Nubie, Capra (Aegoceros) nubiana (17);

- en Afrique du Sud, chez le céphalophe couronné, Sylvicapra grimmia et, en Namibic, chez l'oryx, Oryx gazella (28).

La Cénurose est donc relativement rare chez les Ruminants sauvages.

Comme chez les moutons des zones tropicales, les Cénures se localisent dans le conjonctif intermusculaire, dans le conjonctif sous-cutané, dans les ganglions, la glande thyroide et le cerveau.

4.2. Des Catarhiniens Cynomorphes appartenant à la famille des Cercopithecinae et aux genres Cercopithecus (Cercopithecus mitis et Cercopithecus nictitans nictitans, le Hocheur) et Theropithecus (Theropithecus gelada, le gélada). On connaît actuellement 13 cas mondiaux de Cénurose du singe dont $11 \mathrm{chez}$ le gélada :

- Europe (22).

- U. S. A. (24).

2 fois seulement, les singes atteints venaient d'être importés de l'Est ou de l'Ouest de l'Afrique, les autres étant soit nés sur place, soit entretenus dans des parcs zoologiques depuis de longues années.

Les lésions siègent le plus souvent dans le tissu conjonctif sous-cutané (maxillaire, paroi thoracique, région inguinale), dans le conjonctif intermusculaire (avant-bras, tibia), dans les séreuses (plèvre et péritoine), beaucoup plus 
rarement dans le cerveau et dans la colonne vertébrale. Elles sont souvent de grande taille de 22 à $36 \mathrm{~cm}$ de diamètre) et leur poids peut atteindre $1980 \mathrm{~g}(22)$.

Des essais d'infestation du chien à partir de ces Cénures ont été tentés plusieurs fois avec (24) ou sans succès (22). Le Cestode obtenu est un Taenia serialis dont les segments gravides administrés à des cobayes, à des lapins, à des souris ou à des agneaux n'entraînent pas la formation de Cénures. SCHWARTZ (24) en conclut que ce parasite est une forme de Taenia serialis qu'il nomme Taenia serialis var. theropitheci. On sait aujourd'hui (27) qu'il s'agit de Taenia serialis brauni dont l'existence en Ethiopie, pays d'origine du gélada, est amplement prouvée, ce qui explique pourquoi cet animal est si souvent porteur.

La Cénurose des singes est grave, car, bien souvent, les kystes sont disséminés dans tout l'organisme et l'issue fatale est de règle (dans plus de 60 p. 100 des cas). Néanmoins, lorsqu'ils sont peu nombreux et superficiels, on peut tenter l'exérèse avec de bonnes chances de succès.

4.3. Chez l'Homme, la Cénurose revêt 2 aspects différents :

4.3.1. Une Cénurose cérébrale à Coenurus cerebralis. Le Cestode semble se localiser de façon préférentielle dans les voies d'écoulement du liquide céphalo-rachidien, surtout dans les espaces sous-arachnoïdiens, plus rarement dans les ventricules cérébraux, exceptionnellement dans les hémisphères et dans l'œil. Les kystes sont, la plupart du temps, multiples : ils se présentent comme des grappes de vésicules, petites, libres ou faiblement attachées les unes aux autres dont la paroi est très mince, réduite à la seule membrane germinative. Aussi, les signes cliniques sont-ils très variables : méningite, hypertension intra-crânienne, céphalées, vertiges, agraphie, alexie... Le pronostic est sombre. La mortalité atteint près de 60 p. 100 et, dans les cas favorables, l'opération laisse souvent des séquelles graves.

4.3.2. Une Cénurose à localisation souscutanée ou oculaire.

La première se traduit par la présence de nodules sous-cutanés de 2 à $6 \mathrm{~cm}$ situés sous le fascia, généralement sur le tronc, la région intercostale et la paroi abdominale, beaucoup plus rarement sur le cou, la tête et les membres.
La seconde intéresse la chambre antérieure de l'œil, l'humeur vitrée et la conjonctive.

Le pronostic est favorable: les lésions sont facilement opérables et l'intervention ne laisse pas de traces.

Les Cénures en cause sont Coenurus serialis serialis et Coenurus serialis brauni.

On dénombre actuellement une centaine de cas de Cénurose humaine dont 77 pour l'Afrique seule. Ils se répartissent ainsi :

- Cénurose cérébrale : 31 cas dont 12 en Afrique du Sud (29) et 19 en dehors de l'Afrique (9): 13 en Europe (France, Angleterre, Sardaigne, Espagne), 2 en Russie, 3 aux U. S. A. et au Mexique et 1 au Brésil.

- Cénurose sous-cutanée et oculaire : 48 cas dont 4 (France, Espagne) par Coenurus serialis serialis. Les 44 autres ont pour origine Coenurus serialis brauni et sont tous africains :

- Ruanda et République démocratique du Congo : 11 cas (12) ;

- Kenya : 1 cas (20);

- Uganda : 32 cas $(20,25)$.

- Cas reconnus, mais non publiés de Cénurose cérébrale : 11 en Afrique du Sud et de Cénurose sous-cutanée : 3 en Uganda $(20,25)$.

- Cas où l'identification formelle du parasite n'a pas été possible : 1 cas aux U. S. A. ; 2 cas en Afrique du Sud; 3 cas au Nigeria; 1 cas au Ghana (30) et 1 cas en Afrique centrale (26). La Cénurose rencontrée au Ghana et au Nigeria (Provinces du Nord et Ibadan) est de type souscutané. Le Cestode en cause a d'abord été identifié comme étant Coenurus glomeratus (= Coenurus brauni). Cependant, en Afrique de l'Ouest, la plus grande prudence s'impose, car, sur les exemplaires de WILSON, WAYTE et ADDAE (30), la longueur des crochets laisse supposer qu'il s'agirait plutôt de Coenurus serialis serialis que de Coenurus serialis brauni.

Le cas de Cénurose cérébrale décrit par TRUELLE, HOUTTEVILLE, RICOU et LE BIGOT (26) concerne un homme (*) qui, après avoir passé son enfance en Afrique du Sud et au Mexique, a parcouru, depuis 32 ans, l'Afrique noire, notamment le Tchad, la République populaire du Congo, le Dahomey et la Côte-d'Ivoire, pour se fixer enfin au Gabon. L'ancienneté du séjour en Afrique du Sud et au Mexique permet d'éliminer la possibilité

(*) Communication personnelle. 
d'une infestation dans ces deux pays, infestation qui aurait pu être contractée en Afrique noire où la Cénurose des petits Ruminants à Coenurus cerebralis existe tant au Tchad qu'au Congo.

\section{CONCLUSIONS}

1. De 1954 à 1969,7 cas de Cénurose ont été observés chez les petits Ruminants du Tchad et de la République populaire du Congo dont $1 \mathrm{chez}$ la chèvre et 6 chez le mouton.

Le taux d'infestation est, dans l'une ou l'autre espèce, légèrement supérieur à $0,1 \mathrm{p} .100$.

2. La maladie se traduit par la présence, dans le tissu conjonctif sous-cutané et intermusculaire de l'épaule, de la cuisse et de la paroi thoracique, dans les séreuses et, beaucoup plus rarement, dans le cerveau, de kystes, véritables pseudo-tumeurs qui occasionnent des troubles variés, surtout locomoteurs.

3. Ces Cénures sont comparés avec un parasite de même nature prélevé dans l'encéphale d'un mouton européen.

L'aspect du Cestode, la longueur et la forme des crochets indiquent qu'il s'agit bien de Coenurus cerebralis (Batsch, 1786) Rudolphi, 1808 dont la forme adulte, Taenia multiceps Leske, 1780 vit dans l'intestin de divers Canidés domestiques et sauvages (chacal).

Taenia gaigeri Hall, 1916 et Taenia skrjabini Popov, 1937 qui, à l'état larvaire, provoquent, chez les petits Ruminants des zones tropicales et des steppes d'Asie centrale, une Cénurose sous-cutanée et intermusculaire ne sont que des variétés ou, peut-être, des sous-espèces de Taenia multiceps.

4. Taenia serialis des Carnivores dont le Cénure se localise dans le tissu conjonctif souscutané des Lagomorphes est une espèce qui, sur de simples critères anatomiques ou morphologiques, ne peut être distinguée sûrement de la précédente, ce qui amène certains auteurs à les confondre toutes deux.

Or, du point de vue biologique, il n'a jamais été possible de reproduire la Cénurose du lapin à partir d'anneaux ovigères de Taenia multiceps et, réciproquement, la Cénurose du mouton à partir d'anneaux ovigères de Taenia serialis, comme le montrent des essais récents effectués au Sénégal.

On est donc bien obligé d'admettre la dualité des espèces.

Taenia serialis comprend 2 sous-espèces :

- Taenia serialis serialis (Bailliet, 1863) Verster, 1969 n. comb.

- Taenia serialis brauni (Setti, 1897) Verster, 1969 n. comb. des Carnivores domestiques et sauvages qui, à l'état larvaire, se développe dans le conjonctif sous-cutané desRon geurs (Muridés, Rhyzomyidés, Hystricidés) et des singes dont le gélada (13 cas mondiaux).

5. Actuellement, la répartition de la Cénurose animale sur le continent africain est la suivante :

- Coenurus cerebralis des petits Ruminants et des Bovidés sauvages: Afrique du Sud et Angola, Afrique Centrale (Tchad et République populaire du Congo), Afrique de l'Est et du Nord-Est (Egypte, Soudan, Ethiopie, Kenya), Afrique de l'Ouest (Sénégal) ;

- Coenurus serialis serialis des Léporidés : Afrique de l'Ouest (Sénégal, Dahomey, Ghana), Afrique centrale (Tchad), Afrique du Sud et Angola, Afrique du Nord-Est (Egypte, Ethiopie);

- Coenurus serialis brauni: Afrique de l'Est et du Nord-Est (Egypte, Ethiopie, Kenya, Ruanda, République démocratique du Congo), Afrique du Nord (Tunisie) et Afrique du Sud;

6. La Cénurose est susceptible d'affecter l'Homme. Sur les 100 cas mondiaux connus, plus de 75 p. 100 d'entre eux sont d'origine africaine. La Cénurose cérébrale à Coenurus cerebralis existe en Afrique du Sud et, vraisemblablement, en Afrique centrale.

La Cénurose sous-cutanée et la Cénurose oculaire se rencontrent dans l'Est africain (Ruanda, Kenya, Uganda).

En Afrique de l'Ouest (Nigeria, Ghana), les parasites recueillis n'ont pu être identifiés de façon certaine.

\section{SUMMARY}

\section{Coenurosis of sheep and goats in Central Africa. Human and animal} african coenurosis

The author in Chad and Popular Congo Republic points out the presence of Coenurus cerebralis (Batsch, 1786) Rudolphi, 1808 in the subcutaneous and intermuscular tissues, in the pleural and abdominal cavities and in the brain of sheep and goats. Seven cases were observed between 1954 and 1969. 
In Africa, three Cestodes have been found in domestic and wild Carniyores (Jackal) : Taenia multiceps Leske, 1780 ; Taenia serialis serialis (Bailliet, 1863 ) Verster, $1969 \mathrm{n}$. comb. and Taenia serialis brauni (Setti, 1897) Verster, $1969 \mathrm{n}$. comb. The validity of these species is discussed.

The larval forms are : Coenurus cerebralis of domestic and wild Ruminants which exists not only in South Africa, but also in West, East, North-East and Central Africa ; Coenurus serialis serialis of Rabbits and hares in West, central, South and North-East Africa ; Coenurus serialis brauni of Muridae and Monkeys in North, South and East Africa.

Coenurosis is rarely seen in man. On 100 human cases reported in world, 77 of these occur in Africa. Two separate species are concerned in the infection : Coenurus cerebralls in South Africa and, probably, in Central Africa ; Coenurus serialis brauni in East Africa. The four west african cases are unidentified.

\section{RESUMEN}

La cenurosis de los pequeños rumiantes de Africa Central. Las cenurosis africanas, humanas $\mathbf{y}$ animales

El autor señala la presencia de Coenurus cerebralis (Batsch, 1786) Rudolphi, 1808 en el tejido conjuntivo subcutáneo e intermuscular, las serosas y el cerebro de la oveja y de la cabra en Chad y en República popular de Congo.

En resumidas cuentas, se observaron siete casos entre los 5000 pequeños rumiantes autopsiados de 1954 a 1969.

Estudia tres cestodos parásitos, en Africa, del intestino de los cánidos domésticos y salvajes: Taenia multiceps Leske, 1780, Taenia serialis serialis (Bailliet, 1863) Verster, 1969 n. comb. y Taenia serialis brauni (Setti, 1897) Verster, 1969 n. comb. Se precisan sus afinidades.

Sus formas larvarias son:Coenurus cerebralis de los rumiantes domésticos y salvajes que se encuentra no sólo en Africa del Sur sino tambien en Africa del Oeste, del Este y del Centro ; Coenurus serialis serialis de los leporidos de Africa del Oeste, del Sur y del Nordeste ; Coenurus serialis brauni de los Muridos y de ciertos monos de Africa del Norte, de Africa del Sur y de Africa del Este.

La cenurosis es rara pero 77 p. 100 de los casos observados en el mundo se encuentran en Africa. Se tratan de dos especies diferentes: Coenurus cerebralis en Africa del Sur y verosimilmente en Africa Central; Coenurus serialis brauni en Africa del Este. No se pudo identificar con bastante exactitud los casos ocurridos en Africa del Oeste.

\section{BIBLIOGRAPHIE SOMMAIRE $\left(^{*}\right)$}

1. ABULADZE (K. I.). Essentials of Cestodology. Vol. IV. Taeniata of animals and man and diseases caused by them. Moscou, Acad. U. R. S. S., Lab. Helminth., 1964 et Jerusalem, Israel program Sci. Transl., 1970.

2. BAILLIET (C.). Recherches sur un Cysticerque polycéphale du Lapin et sur le ver qui résulte de sa transformation dans l'intestin du Chien. Mém. Acad. Sci. nat., Toulouse 1863, 6s (I): 452-482.

3. BERGEON (P.). Veterinary Parasitology Survey. Report Government of Ethiopia. Rome F. A. O., 1968, no 2458, 1-38.

4. BHADURI (N. V.), MAPLESTONE (P. A.). Variations in Taenia gaigeri Hall, 1916. Rec. Indian Mus., 1940,42 : 431-435.

5. CLAPHAM (P. A), PETERS (B. G.). The differentiation of Coenurus species by hook measurements. J. Helminth., 1941, 19 (3/4) : 75-84.

6. CLAPHAM (P. A.). On two new Coenuri from Africa and a note on the development of the hook. J. Helminth., 1942, 20 (1/2) : 25-31.

(*) Une Bibliographie beaucoup plus complète (150 titres environ), excluant toutefois la Cénurose du mouton et la Cénurose des Léporidés, est à la disposition des lecteurs intéressés qui pourront l'obtenir gratuitement en s'adressant au centre de Documentation de l'I. E. M. V. T., 10, rue Pierre-Curie, 94700 MaisonsAlfort, France.
7. CLAPHAM (P. A.). On identifying Multiceps sp. by measurements of the large hooks. $J$. Helminth., 1942, $20(1 / 2): 31-40$.

8. ESCH (G. W.), SELF (J. T.). A critical study of the taxonomy of Taenia pisiformis Bloch, 1780, Multiceps multiceps Leske, 1780 and Hydatigera taeniaeformis Batsch, 1786. Parasitology., 1965, 51 (6): 932-937.

9. EUZEBY (J.). Zoonotic Metacestodiasis. Clinical and experimental studies. San Francisco, Academic Press, 1974, 151-178.

10. EZZAT (M. A. E.). The occurrence of Multiceps gaigeri Hall, 1916 in subcutaneous connective tissue of Sudanese sheep and Nubian Ibex. Egypt. Minist. Agric. vet. Serv., 1944 (238) : 1-6.

11. FAIN (A.). Cénurose chez l'homme et chez les animaux due à Taenia brauni, Setti au Congo Belge et au Ruanda-Urundi, I. La Cénurose chez les animaux sauvages avec existence de localisations générales. Annls Soc. belge Méd. trop., 1956, 36 (5 bis) : 673-677.

12. FAIN (A.), DENISOFF (N), HOMANS (L.), QUESTIAUX (G.), VAN LAERE (L.), VINCENT (M.). Cénurose chez l'homme et les animaux due à Taenia brauni, Setti au Congo Belge et au RuandaUrundi. II. Relation de huit cas humains. Annls. Soc. belge Méd. trop., 1956, 36 (5 bis) : 679-696.

13. GAIGER (S. H.). Coenurus serialis found in two Goats in India. J. trop. vet. Sci., Calcutta, 1907, 2 (3) : 316-321.

14. HALL (M. C.). The gig parasite and allied species of the Cestode genus Multiceps. I. Historical review. U. S. Dept. Agric. Bur. Anim. Ind., 1910 (125), I : 1-68. 
15. HALL (M. C.). A new and economically important Tapeworm Multiceps gaigeri from the dog. J. am. vet. med. Ass., 1916, 50 (3) : 214-223.

16. MOREIRA (F.), SERRANO (H.). Fauna helminthologica dos animais domesticos de Angola. Anass. Serv. vet. Angola, 1962, 20 : 1-85.

17. NAGATY (H. F.), EZZAT (M. A. E.). On the identity of Multiceps multiceps Leske, 17-80, Multiceps gaigeri Hall, 1916 and Multiceps serialis Gervais, 1845 , with a review of these and similar forms in man and animals. Proc. helminth. Soc. Wash., 1946, 13 (2) : 33-44.

18. NELSON (G. S.), PESTER (F. R. N.), RICKMAN (R.). The significance of wild animals in the transmission of Cestodes of medical importance in Kenya. Trans. R. Soc. trop. Med. Hyg., 1965, 59 (6) : 507-524.

19. RAMADAN (R. O.), MAGZOUB (M.), ADAM (S. E. 1.). Clinicopathological effects on a Sudanese goat following massive natural infection with Coenurus gaigeri cysts. Trop. anim. Hith. Prot, 1973, 5 (3) : 196-199.

20. RAPER (A. B.), DOCKERAY (C. C.). Coenurus cysts in man : five cases from East africa. Ann. trop. Med. Parasit., 1956, 59 (6) : 502-524.

21. Rapport annuel du Laboratoire de l'Elevage, Dakar Hann, Sénégal, 1969.

22. RODHAIN (J.), WANSON (M.). Un nouveau cas de Coenurose chez le babouin, Theropithecus gelada, Ruppell. Riv. Parassit., 1954, 15 (4) ; 613-620.

23 ROUND (M. C.). The Helminth parasites of domesticated animals in Kenya. J. Helminth., 1962, 36 (4) : 375-449.

24. SCHWARTZ (B.). A subcutaneous tumour in a Primate caused by tapeworm larvae experimentally reared to maturity in dogs. J. agric. Res., 1927, 35 (5) : 471-480.

25. TEMPLETON (A. C.). Anatomical and geographical location of human Coenurus infection. Trop. geogr. Med., 1971, 23 (1) : 105-108.

26. TRUELLE (J. L.), HOUTTEVILLE (J. P.), RICOU (Ph.), LE BIGOT (P.). Cénurose cérébrale intraventriculaire. Etiologie rare de Ménıngite chronique. Nouv. Presse Méd., 1974, 3 (18) : 1151-1153.

27. VERSTER (A.). A taxonomic revision of the genus Taenia Linnaeus, $1758 \mathrm{~s}$. Str. Onderstepoort $J$, vet. Res., 1969, 36 (I) : 3-58.

28. VERSTER (A.), BEZUIDENHOUT (J. D.). Taenia multiceps larva from a Gemsbok. Onderstepoort $J$. vet. Res., 1972, 39 (2) : 123.

29. WAINWRIGHT (J.). Coenurus cerebralis and racemose cysts of the brain. J. Path. Bact., 1957, 73 (2): 347-354.

30. WILSON (V. C. L.), WAYTE (D. M.), ADDAE (R. O.). Human Coenurosis. The first case from Ghana. Trans. R. Soc. trop. Med. Hyg., 1972, 66 (4) : 611-623. 